\title{
The usefulness of C-reactive protein and neutrophil-to-lymphocyte ratio for predicting the outcome in hospitalized patients with liver cirrhosis
}

\author{
Jung Hyun Kwon ${ }^{1}$, Jeong Won Jang ${ }^{2 *}$, Young Woon Kim¹, Sung Won Lee ${ }^{3}$, Soon Woo Nam', Dongwook Jaegal',
} Seungok Lee ${ }^{4}$ and Si Hyun Bae ${ }^{2}$

\begin{abstract}
Background: The role of clinical parameters such as systemic inflammatory response syndrome (SIRS) criteria in predicting the infection remains unclear in cirrhosis patients. The aim was to evaluate the usefulness of inflammatory markers including C-reactive protein (CRP) and the neutrophil-to-lymphocyte ratio (NLR) for diagnosis of infection and predicting the outcomes in hospitalized cirrhotic patients.

Methods: The study included 184 cirrhotic patients consecutively hospitalized from 2011 to 2012. The presence of overt infection and survival was evaluated. CRP concentration, NLR, Model for End-Stage Liver Disease (MELD) score and the presence of SIRS were assessed.

Results: The main cause of admission was uncontrolled ascites (36.4\%), followed by varix bleeding (23.9 \%), and hepatic encephalopathy (13.6\%). Fifty-eight patients (31.5\%) had overt infection during hospitalization and thirty-two patients (17.4\%) expired during the follow up period (median 38 months). Ninety-two patients (52.2\%) fulfilled the SIRS criteria and among them, only 32 patients (38.5 \%) had the overt infection. For diagnose of the infection, baseline CRP concentration was a significant factor compared to the presence of SIRS (odds ratio 1.202, $P=0.003$ ). For predicting one-month short-term survival, MELD score, NLR and WBC count were significant factors but in Child-Pugh class C patients, NLR was only an independent factor.
\end{abstract}

Conclusions: CRP was a significant indicator of infection in hospitalized cirrhotic patients and a NLR was a useful predictor of 1-month survival, particularly in Child-Pugh class C patients. This study suggests that the inflammatory markers such as CRP and NLR can help identify cirrhotic patients at risk of unfavorable outcomes.

Keywords: C-reactive protein, Liver cirrhosis, Infection, Neutrophil-to-lymphocyte ratio, Survival

\section{Background}

In cirrhotic patients, the severity of liver disease is an important factor influencing the risk of mortality $[1,2]$. Systemic inflammation occurs frequently in patients with advanced cirrhosis [2,3] and might be associated with a negative outcome [4]. Bacterial infections can lead to both a longer hospital stay and increased mortality in patients with cirrhosis [5-7]. Previous studies have

\footnotetext{
* Correspondence: garden@catholic.ac.kr

${ }^{2}$ Department of Internal Medicine, Seoul St. Mary's Hospital, The Catholic University of Korea, 222 Banpo-daero, Seocho-gu, Seoul, South Korea Full list of author information is available at the end of the article
}

reported that bacterial infections are present or develop during hospitalization in around the one-third of hospitalized cirrhotic patients $[3,6,8]$. Systemic inflammatory response syndrome (SIRS) has been strongly suggestive of the presence of infection and association with poor outcomes $[2,4,9]$. However, the specific characteristics of cirrhotic populations, such as hypersplenism, hyperventilation associated with hepatic encephalopathy and hepatorenal syndrome, increase the difficulty of identifying episodes and the use of beta-blockers may modify the clinical and biochemical parameters used to identify SIRS [10]. Thus, the presence of overt infection cannot 
be excluded in patients with cirrhosis who do not meet the SIRS criteria. Although the liver function markers such as the Model for End-Stage Liver Disease (MELD) score is a good factor for survival, it has some limitations to reflect the acute status of hospitalized cirrhotic patients. Hospitalization in cirrhotic patients would be required at the decompensated events or infection and so on. This is why a convenient and easy-to-measure parameter is needed for predicting the outcomes in the unstable patients group.

C-reactive protein (CRP), which is synthesized in the liver, has been identified as an inflammatory marker [11]. We previously reported the usefulness of CRP in predicting short-term mortality in patients with hepatocellular carcinoma (HCC), and outcomes after liver transplantation [12, 13]. CRP is also an acute phase reactant that is suggested to be a marker of the presence of bacterial infection [14]. The neutrophil-to-lymphocyte ratio (NLR) has been suggested as a marker of systemic inflammation and shows the relationship between two different immune pathways [15]. The neutrophil count reflects ongoing inflammation, whereas the lymphocyte count represents the immune regulatory pathway. The NLR has been used to predict outcomes in patients with cancer and cardiac disease $[15,16]$. It was recently reported to predict the outcomes in patients with $\mathrm{HCC}$, nonalcoholic fatty liver disease, and liver transplantation $[12,17,18]$, Although clinical risk factors of bacterial infection, such as advanced liver diseases or gastrointestinal bleeding, are well characterized [6, 8, 19-21], data on both CRP and NLR as serologic markers are scarce in such patients with advanced diseases. The aim of this study was to investigate whether CRP and NLR could significantly improve the diagnostic accuracy of infection and advance the predictive power of short-term mortality in a large consecutive cohort of patients, who were hospitalized due to hepatic events.

\section{Methods}

\section{Patients}

This was a retrospective observational cohort study performed at a university hospital. The study included a cohort of 184 consecutive cirrhotic patients hospitalized in a tertiary center from September 2011 to September 2012. The diagnosis of cirrhosis was based on liver biopsy or clinically when the patients had at least two of the following three criteria: an inhomogeneous hepatic surface with splenomegaly or portal hypertension on radiological findings; platelet count $<100,000 / \mathrm{mm} 3$ or variceal changes on endoscopy [22]. The exclusion criteria were a previous diagnosis of HCC or extrahepatic malignancy and an elective admission to evaluate the suspicion of HCC. This study was approved by the Office of Human Research Protection Program,
Catholic Medical Centre (CMC OHRP, reference number; OC13RISI0127) and the need for consent from participants was waived by the CMC OHRP. This study was also in accordance with the Helsinki Declaration of 1975.

The main clinical events for hospitalization such as uncontrolled ascites, variceal bleeding, hepatic encephalopathy, or other signs of hepatic deterioration were reviewed carefully and evaluated. When there was more than one main clinical event at admission, the criteria used to define the main cause of admission were the following: (a) Whenever variceal bleeding was coincident with other major complications, it was considered the main cause of admission, (b) In patients with hepatic encephalopathy and other events, the former was considered the main cause of admission, (c) All the types of infection were counted and analyzed even if it was a single present or co-exist with other clinical events [3].

At admission, patients routinely underwent a physical examination, laboratory tests, and X-ray of the chest and abdomen. Laboratory tests included blood chemistry and blood cell counts including CRP concentration and ascitic/pleural fluid cell counts. Serum CRP level was measured as high-sensitivity CRP by an immunoturbidimetric assay using the C-Reactive Protein, High Sensitivity reagent (Beckman Coulter, Inc., Fullerton, CA, USA; limit of detection, $0.08 \mathrm{mg} / \mathrm{L}$ ). The NLR was calculated by dividing the neutrophil count by the lymphocyte count. All the hospitalized patients were administered empiric broad-spectrum antibiotics (ceftriaxone or ciprofloxacin) after initial laboratory test and physical examination, and the regimen was modified or stopped according to the results of the cultures or the infection [7].

During hospitalization, cultures of blood, urine, ascites, pleural effusion, and sputum or swabs were taken when an infection was suspected. The severity of liver disease was assessed according to the Child-Pugh class and the MELD score. Physical examination and laboratory tests to measure blood cell counts and chemistry were daily performed. Depending on the creatinine level, abdominal ultrasonography or computed tomography was performed if needed.

\section{Definition of systemic inflammation and infection}

SIRS was assessed according to the recommendations of the American College of Chest Physicians/Society of Critical Care Medicine Consensus Conference [23]. Patients were considered to have SIRS if they fulfilled at least two of the following criteria: (a) core temperature of $>38^{\circ} \mathrm{C}$ or $<36{ }^{\circ} \mathrm{C}$; (b) heart rate of $>90$ beats $/ \mathrm{min}$; (c) respiratory rate of $>20$ breaths/min; partial carbon monoxide pressure $(\mathrm{PaCO} 2) \leq 32 \mathrm{mmHg}$ or the need of mechanical ventilation or (d) white blood cell (WBC) 
count of $>12,000 / \mathrm{mm}^{3}$ or $<4000 / \mathrm{mm}^{3}$, or differential count showing $>10 \%$ immature polymorphonuclear neutrophil cells (PMNCs).

The most common bacterial infection in cirrhosis is known to be spontaneous bacterial peritonitis (SBP), urinary tract infection, followed in frequency by pneumonia, and bacteremia [3, 6, 24]. For this evidence, diagnosis of SBP was considered as an ascitic fluid PMNC count $>250 / \mathrm{mm}^{3}$. Urinary tract infection was diagnosed as a urinary WBC count $>10$ cells per high-power field or a positive urine culture [3]. Pneumonia was diagnosed if there was radiographic evidence of pulmonary infiltration associated with purulent sputum. Bacteremia was diagnosed as a positive blood culture in the absence of any recognized source of infection [3, 4]. Other infections such as cellulitis, biliary infection, and enterocolitis were diagnosed according to clinical, radiological, and bacteriologic data. Community-acquired infections were defined as those that were recognized before admission or within the first $48 \mathrm{~h}$, and hospital-acquired infections or delayed infections were diagnosed after this period [3].

\section{Statistical analysis}

SPSS version 18 (SPSS Inc., Chicago, IL, USA) was used to analyze the data. The data was expressed as the mean \pm SD or as the median and range. Logistic regression analysis was performed to assess significant differences in predicting 1-month survival and the presence of infection. Overall survival was calculated from the date of admission to the date of death or the last follow-up. For patients undergoing liver transplantation, followup was censored at the time of transplantation. The factors affecting the survival rate were identified using Cox's proportional-hazard model.

\section{Results}

\section{Baseline characteristics of hospitalized cirrhotic patients} with and without infection

One hundred eighty-four patients were included in the study (Table 1): 125 (67.9 \%) men with a mean age of $56.7 \pm 11.7$ years. The main clinical events for hospitalization were uncontrolled ascites in 67 patients (36.4 \%), variceal bleeding in 44 patients $(23.9 \%)$, and hepatic encephalopathy in 25 patients (13.6\%), alcoholic withdrawal seizure in 6 patients (3.2\%). Most patients had severe liver disease of Child-Pugh class B or C $(82.5 \%)$ and average MELD score was $12.2 \pm 7.5$.

Fifty-eight of 184 patients $(31.5 \%)$ had overt infection during admission, of whom 49 (85\%) patients were diagnosed a community acquired infection. The most common infections were urinary tract infection $(n=21$, $36.2 \%)$ followed by pneumonia/bacteremia ( $n=9 / 9$,

Table 1 Baseline characteristics of hospitalized cirrhotic patients with or without infection

\begin{tabular}{|c|c|c|c|}
\hline & $\begin{array}{l}\text { Patients with infection } \\
(n=58,31.5 \%)\end{array}$ & $\begin{array}{l}\text { Patients without infection } \\
(n=126,68.5 \%)\end{array}$ & $P$ values \\
\hline Age (year-old) & $60.2 \pm 12.4$ & $55.0 \pm 11.0$ & 0.005 \\
\hline Sex (male, \%) & $33(56.9 \%)$ & $92(73 \%)$ & 0.030 \\
\hline $\mathrm{HBV} / \mathrm{HCV} / \mathrm{Alc} /$ others & $20 / 3 / 27 / 8$ & $31 / 11 / 82 / 2$ & 0.002 \\
\hline Child-Pugh classification (A/B/C) & $11 / 26 / 21$ & $23 / 59 / 44$ & 0.969 \\
\hline MELD score & $13.2 \pm 8.0$ & $11.9 \pm 19.1$ & 0.215 \\
\hline Meet the criteria of SIRS (\%) & $37(63.8 \%)$ & $59(46.9 \%)$ & 0.032 \\
\hline Body temperature & $36.8 \pm 0.9$ & $36.3 \pm 0.5$ & 0.000 \\
\hline Neutrophil to lymphocyte ratio & $8.3 \pm 10.1$ & $4.9 \pm 6.8$ & 0.027 \\
\hline WBC count $\left(/ \mathrm{mm}^{3}\right)$ & $8971.7 \pm 5340.8$ & $7362.5 \pm 4668.9$ & 0.100 \\
\hline Neutrophil count (\% of WBC) & $71.8 \pm 13.7$ & $65.1 \pm 14.0$ & 0.003 \\
\hline Lymphocyte count (\% of WBC) & $17.8 \pm 10.9$ & $23.0 \pm 12.1$ & 0.004 \\
\hline CRP (mg/L) & $34.1 \pm 49.2$ & $11.9 \pm 19.1$ & 0.002 \\
\hline \multicolumn{4}{|l|}{ Baseline laboratory data } \\
\hline Platelet count $\left(/ \mathrm{mm}^{3}\right)$ & $116.8 \pm 70.0$ & $105.2 \pm 55.3$ & 0.272 \\
\hline Albumin (g/dL) & $3.0 \pm 0.7$ & $3.0 \pm 0.6$ & 0.794 \\
\hline Total bilirubin (mg/dL) & $3.3 \pm 3.1$ & $3.9 \pm 4.6$ & 0.373 \\
\hline Prothrombin time (INR) & $1.5 \pm 0.6$ & $1.5 \pm 0.5$ & 0.937 \\
\hline Creatinine (mg/dL) & $1.1 \pm 0.9$ & $0.9 \pm 0.7$ & 0.118 \\
\hline $\mathrm{Na}(\mathrm{mEq} / \mathrm{L})$ & $132.2 \pm 18.3$ & $134.9 \pm 12.5$ & 0.223 \\
\hline
\end{tabular}

MELD model for end-stage liver disease, SIRS systemic inflammatory response syndrome, CRP C-reactive protein, INR international normalized ratio Differences assessed using the Mann-Whitney $U$ test for numerical variables and chi-test for categorical variables 
$15.5 / 15.5 \%)$, SBP $(n=6,10.3 \%)$, cellulitis/biliary tract infection/enterocolitis $(n=3 / 3 / 3,5.2 / 5.2 / 5.2 \%)$ and others $(n=4,6.9 \%)$. The patients with infection were old and female dominant compared to those without infection. However, the alcohol related cirrhosis in the patients with infection was less than that in the patients without infection although it was the most common cause of underlying liver cirrhosis (Table 1). The number of patients who met the SIRS criteria at admission was 96 (52.2 \%). The patients with infection fulfilled the SIRS criteria significantly more than those without infection (63.8 \% versus $46.9 \%, P=0.032$ ). Among the SIRS criteria, the body temperature in patients with infection was significantly higher than those without infection. Otherwise, heart rate, respiration rate and $\mathrm{PaCO}_{2}$ levels did not differ between patients with and without infection. The platelet counts and any other liver panel tests were not different depending on the presence of infection.

\section{Baseline CRP level predicts the infection in hospitalized cirrhotic patients}

The levels of CRP in patients with infection were significantly higher than those without infection (Fig. 1a). In addition, NLR was significantly higher in patients with infection than those without, in whom WBC counts were not different between the two groups (Fig. 1a). Specifically, the neutrophil count was higher and the lymphocyte count was lower in cirrhotic patients with infection than those without (Table 1). However, the MELD score did not differ depending on infection. Nine out of 45 patients developed delayed infection after admission, whose CRP and NLR did not differ compared to those with community-acquired infection.

For predicting infection using the logistic regression analysis, old age, female gender, non-alcohol related liver cirrhosis, the presence of SIRS, high level of CRP and NLR were significant factors (Table 2). In the multivariate analysis, female gender and high baseline CRP were predictable risk factors for the infection in hospitalized cirrhotic patients.

With regard to SIRS, CRP showed the trend to increase in patients showing the SIRS (Fig. 1b). NLR and MELD score in the patients who met the SIRS criteria were significantly higher than those who did not (Fig. 1b). For our cohort, the level of the CRP and the NLR positively correlated with the Child-Pugh class but it didn't show the statistical significance (Fig. 1c).

\section{Neutrophil to lymphocyte ratio predicts the short-term survival in hospitalized cirrhotic patients}

Thirty-two patients expired during the mean follow up period $429.3 \pm 302.4$ days, of whom eight patients expired within one month of admission. Patients with high MELD had significantly poor survival regardless of the presence of infection or SIRS $(P=0.000)$. However, for predicting one-month survival after the hospitalization, the baseline NLR as well as MELD score and WBC count was a significant factor (Table 3). Especially, in the patients with Child-Pugh class $\mathrm{C}$, a high NLR was an independent predictor for the one-month survival after admission (Table 3).

\section{Discussion}

The hospitalized cirrhotic patients suffer from acute decompensated events underlying chronic inflammation status. The present study revealed that CRP and NLR are useful diagnostic markers for infection compared to the SIRS in hospitalized cirrhosis patients. In addition, the baseline NLR predicted the one-month survival as did the MELD score in hospitalized cirrhosis patients especially with Child-Pugh class $\mathrm{C}$.

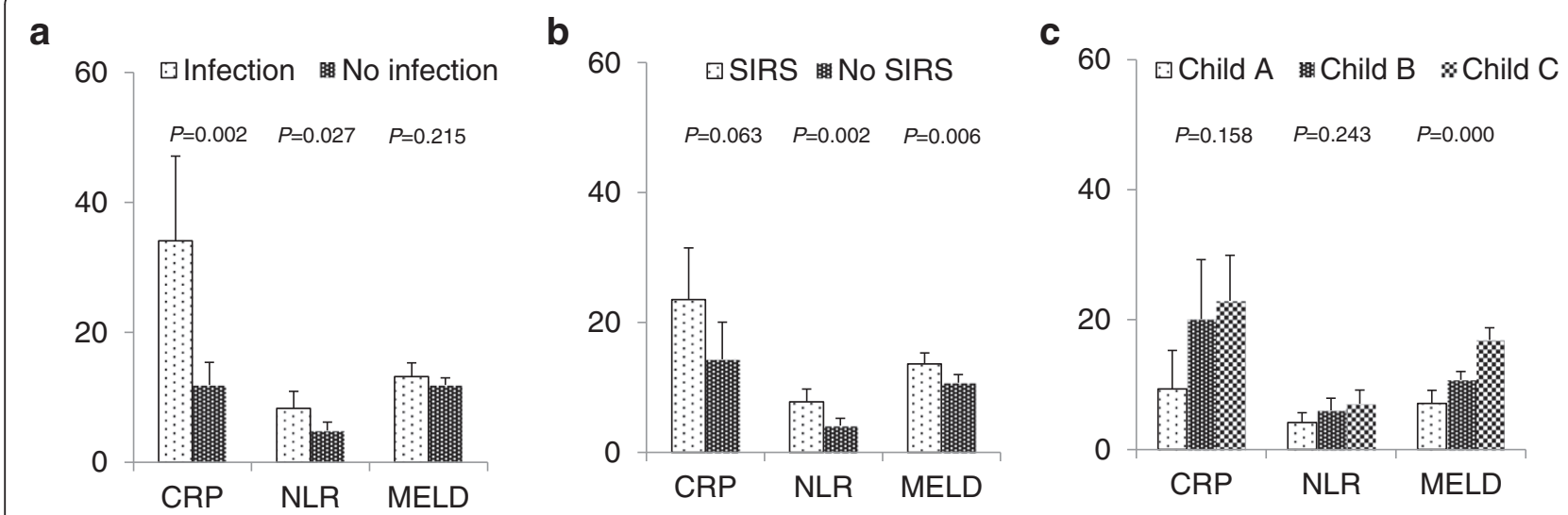

Fig. 1 a CRP level and NLR were significantly higher in infected patients than uninfected patients. $\mathbf{b}$ NLR and MELD score in patients with SIRS were significantly higher than in those without SIRS. c CRP level, NLR and MELD score increased with progressing Child-Pugh class, but MELD score only reached the statistical difference. Boxes and bars show the means and $95 \%$ confidence intervals. CRP, C-reactive protein; NLR, neutrophil to lymphocyte ratio; MELD, model for end-stage liver disease; SIRS, systemic inflammatory response syndrome 
Table 2 Logistic regression analysis for predicting the infection in hospitalized cirrhotic patients

\begin{tabular}{|c|c|c|c|c|}
\hline \multirow{3}{*}{$\begin{array}{l}\text { Baseline } \\
\text { variables }\end{array}$} & \multicolumn{4}{|c|}{ Logistic regression analysis } \\
\hline & \multirow{2}{*}{$\begin{array}{l}\text { Univariate analysis } \\
P \text { values }\end{array}$} & \multicolumn{3}{|c|}{ Multivariate analysis } \\
\hline & & $P$ values & Odds ratio & $95 \% \mathrm{Cls}$ \\
\hline Age & 0.006 & 0.120 & 1.024 & $0.994-1.056$ \\
\hline Female & 0.031 & 0.042 & 2.284 & $1.030-5.065$ \\
\hline Non-alcoholic LC & 0.018 & 0.162 & 1.727 & $0.803-3.717$ \\
\hline Child-Pugh score & 0.976 & & & \\
\hline MELD score & 0.216 & & & \\
\hline SIRS & 0.034 & 0.316 & 1.454 & $0.700-1.034$ \\
\hline CRP & 0.001 & 0.003 & 1.202 & $1.007-1.304$ \\
\hline NLR & 0.019 & 0.181 & 1.034 & $0.985-1.086$ \\
\hline WBC & 0.086 & & & \\
\hline
\end{tabular}

$C l$ confidence intervals, $L C$ liver cirrhosis, MELD model for end-stage liver disease, SIRS systemic inflammatory response syndrome, CRP C-reactive protein, NLR neutrophil-to-lymphocyte ratio

As expected, $82.5 \%$ of the patients in this study had severe liver disease with Child-Pugh class B or C, and over half of patients (52.2\%) had SIRS at inclusion. We know that the patients with advanced cirrhosis have a spontaneous increased proinflammatory response compared with noncirrhotic patients because of an imbalance between proinflammatory (enhanced) and antiinflammatory (inhibited) signaling pathways in immune cells $[2,25,26]$. However, the specific characteristics of cirrhotic patients increase the difficulty of identifying SIRS and the presence of SIRS itself do not directly diagnose the infection. Infection was a severe but frequent (20-60\%) complication with decompensated cirrhosis although frequently asymptomatic, and accounted for increased mortality [3, 6]. In present study, $46.8 \%$ of patients without infection also had SIRS although the patients with infection had more SIRS than those without infection. In a previous study [27], $46 \%$ of infected patients with cirrhosis did not have SIRS. Taken together, not all infected patients with cirrhosis develop SIRS. The authors focused on new prognostic factors that could reveal acute status such as infection in addition to standard MELD score and SIRS.

In the present study, CRP level was an independent predictor of infection compared to the SIRS and MELD scores. CRP is an acute-phase reactant synthesized by hepatocytes in response to inflammation and regulated by proinflammatory cytokines [28]. In our previous report [29], CRP level was an important factor for survival, and correlated with unfavorable tumor characteristics of HCC. The merit of CRP concentration is a simple marker that can be frequently checked in admitted patients. In another reports, CRP levels were higher in patients with SIRS, infection, or alcoholic hepatitis $[8,14,30]$ and CRP was the most useful diagnostic marker of infection compared with other acute phase reactants such as the procalcitonin, lipopolysaccharidebinding protein, sCD14 [14]. In addition, CRP level was reported associated with a lower response rate to antibiotics, a higher mortality rate in cirrhosis patients with SBP [31]. The present study consists that CRP levels increased in patients with infection, SIRS and worse liver function but the most distinguishable increase was shown in patients with infection. Taken together, our study and previous studies suggest that the CRP may be a surrogate marker for the early identification of infection in hospitalized cirrhotic patients $[8,14,30,31]$.

NLR increased in patients with infection and predicted the short-term outcome in hospitalized cirrhotic patients. An increased WBC count might mean the presence of infection, but cirrhotic patients usually have low

Table 3 Logistic regression analysis for predicting the one-month short survival in the hospitalized cirrhotic patients

\begin{tabular}{|c|c|c|c|c|c|c|c|}
\hline \multirow{4}{*}{$\begin{array}{l}\text { Baseline } \\
\text { variables }\end{array}$} & \multirow{4}{*}{$\begin{array}{l}\text { Univariate analysis } \\
\text { Total patients } \\
P \text { values }\end{array}$} & \multicolumn{6}{|l|}{ Multivariate analysis } \\
\hline & & \multirow{2}{*}{\multicolumn{3}{|c|}{$\begin{array}{l}\text { Child-Pugh A and B patients } \\
(n=119)\end{array}$}} & \multirow{2}{*}{\multicolumn{3}{|c|}{$\begin{array}{l}\text { Child-Pugh C patients } \\
(n=65)\end{array}$}} \\
\hline & & & & & & & \\
\hline & & $P$ values & OR & $95 \% \mathrm{Cls}$ & $P$ values & OR & $95 \% \mathrm{Cls}$ \\
\hline Age & 0.571 & & & & & & \\
\hline Female & 0.230 & & & & & & \\
\hline Alcoholic LC & 0.893 & & & & & & \\
\hline MELD score & 0.001 & 0.161 & 0.610 & $0.828-3.131$ & 0.159 & 1.130 & $0.954-1.338$ \\
\hline CRP & 0.122 & & & & & & \\
\hline NLR & 0.005 & 0.269 & 0.446 & $0.106-1.866$ & 0.022 & 1.246 & $1.032-1.505$ \\
\hline WBC & 0.011 & 0.169 & 1.001 & $1.000-1.001$ & 0.496 & 1.000 & $1.000-1.000$ \\
\hline SIRS & 0.997 & & & & & & \\
\hline Infection & 0.657 & & & & & & \\
\hline
\end{tabular}

$O R$ odds ratio, $C$ confidence intervals, $L C$ liver cirrhosis, MELD model for end-stage liver disease, CRP C-reactive protein, NLR neutrophil-to-lymphocyte ratio, SIRS systemic inflammatory response syndrome 
WBC counts due to hypersplenism and patients with alcoholic cirrhosis show high WBC count even without infection. In the present study, the simple WBC counts were not different between infection and no infection group. However, neutrophil count in the infection group was higher than no infection group and lymphocyte count in the infection group was lower than no infection group. This suggests that NLR is a more powerful predictor of infection than simple WBC counts. The early hyperdynamic phase of infection is characterized by a proinflammatory state, which is associated with suppression of neutrophil apoptosis [32] and increased lymphocyte apoptosis in the thymus and spleen [33]. Recently, the neutrophil dysfunction was reported even in the stable cirrhosis patients [34] and predicted outcomes in cirrhosis and alcohol hepatitis [35]. It might be due to the deranged phagocytic activity of opsonized bacteria of neutrophil in advanced cirrhosis. In the present study, neutrophil also showed the significant difference between infection and no infection group. Until now, there is little about the diagnostic power of NLR for infection in liver cirrhosis even though there are some reports about the role of NLR in infection among patients with acute exacerbation of chronic obstructive pulmonary disease and in emergency department [36, 37]. Therefore, NLR can be a helpful marker for identifying infection in hospitalized cirrhotic patients in addition to CRP.

Accumulating data have shown that NLR may be the outcome predictor of several cancers, cardiac disease and even in liver disease such as HCC, non-alcoholic steatohepatitis, liver transplantation waiting patients [12, 15-18]. Recently, pretreatment NLR was reported to be associated with the prognosis of patients with acute on chronic hepatitis B liver failure [38]. However, there has been not yet clear explanation how elevated NLR would be responsible for liver cirrhosis patients. As previously mentioned, in cirrhotic patients, immune and inflammatory systems are activated and inflammatory markers, such as interleukin- 6 and tumor necrosis factor$\alpha$, have been found to be elevated [39]. Since neutrophil could also suppress $\mathrm{T}$ cell activation through the production of arginase, nitric oxide and reactive oxygen species [40], it induces depletion of lymphocyte-mediated immune response. Therefore, elevated NLR might be a poor prognostic marker in cirrhosis patients. In a previous report in which the stable cirrhosis patients without infection or HCC were enrolled, the NLR predicted survival at 12, 24, and 36 months [41]. By contrast, our patients mostly showed decompensation at admission and NLR could predict one-month survival especially in patients with Child-Pugh class $C$. The overall survival of cirrhotic patients is influenced by many factors such as liver function and the presence of life-threatening episodes. Therefore, the MELD and Child-Pugh scores could predict the overall survival independent of the presence of infection. The NLR reflects the interrelationship between the lymphomononuclear and neutrophilic arms of the process of inflammation through complex cytokine interactions [41]. Thus, the NLR is an indicator of the overall inflammatory status of the body. The NLR was a better predictor of 1month survival than 3- or 6-month survival (data not shown). These findings suggest that NLR reflects the current inflammatory status at admission and thus may be useful for predicting short-term survival for 1 month even in Child-Pugh class $C$ patients whose liver function is poor. In our previous report, NLR was significantly related to underlying hepatic reserve as well as tumor burden [12]. Thus, NLR might be helpful to consider who need the early liver transplantation in addition MELD score.

\section{Conclusions}

In conclusion, CRP and NLR are helpful diagnostic markers of infection in hospitalized cirrhotic patients. In Child-Pugh class C patients, an elevated NLR predicted a poor 1-month survival. Therefore, hospitalized cirrhotic patients with an elevated CRP concentration and NLR should be monitored carefully for the presence of infection. In addition to the classical MELD score, NLR may be a useful predictor of the short-term mortality in hospitalized cirrhotic patients.

\section{Abbreviations \\ SIRS: Systemic inflammatory response syndrome; MELD: Model for End-Stage Liver Disease; CRP: C-reactive protein; HCC: Hepatocellular carcinoma; NLR: Neutrophil-to-lymphocyte ratio; PaCO2: Partial carbon monoxide pressure; WBC: White blood cell; PMNC: Polymorphonuclear neutrophil cell; SBP: Spontaneous bacterial peritonitis.}

\section{Competing interests}

The authors declare that they have no conflict of interest.

\section{Authors' contributions}

JHK and JWJ planned the study design and analyzed and interpreted the data. JHK and JWJ drafted the manuscript. YWK, SWL and SWN enrolled and treated patients and acquired the data. DJ and SL performed the laboratory works. All authors had access to the study data and reviewed and approved the final manuscript

\section{Author details}

'Department of Internal Medicine, Incheon St. Mary's Hospital, The Catholic University of Korea, 56 Dongsu-ro, Bupyeong-gu, Incheon, South Korea. 2Department of Internal Medicine, Seoul St. Mary's Hospital, The Catholic University of Korea, 222 Banpo-daero, Seocho-gu, Seoul, South Korea.

3 Department of Internal Medicine, Bucheon St. Mary's Hospital, The Catholic University of Korea, 327 Sosa-ro, Wonmi-gu, Bucheon-si, Gyeonggi-do, South Korea. ${ }^{4}$ Department of Laboratory Medicine, Incheon St. Mary's Hospital, The Catholic University of Korea, 56 Dongsu-ro, Bupyeong-gu, Incheon, South Korea.

Received: 7 May 2015 Accepted: 15 October 2015

Published online: 23 October 2015

\section{References}

1. Deschenes M, Villeneuve JP. Risk factors for the development of bacterial infections in hospitalized patients with cirrhosis. Am J Gastroenterol. 1999;94(8):2193-7. 
2. Thabut D, Massard J, Gangloff A, Carbonell N, Francoz C, Nguyen-Khac E, et al. Model for end-stage liver disease score and systemic inflammatory response are major prognostic factors in patients with cirrhosis and acute functional renal failure. Hepatology. 2007;46(6):1872-82.

3. Fernandez J, Navasa M, Gomez J, Colmenero J, Vila J, Arroyo V, et al. Bacterial infections in cirrhosis: epidemiological changes with invasive procedures and norfloxacin prophylaxis. Hepatology. 2002;35(1):140-8.

4. Cazzaniga M, Dionigi E, Gobbo G, Fioretti A, Monti V, Salerno F. The systemic inflammatory response syndrome in cirrhotic patients: relationship with their in-hospital outcome. J Hepatol. 2009;51(3):475-82.

5. Caly WR, Strauss E. A prospective study of bacterial infections in patients with cirrhosis. J Hepatol. 1993;18(3):353-8.

6. Borzio M, Salerno F, Piantoni L, Cazzaniga M, Angeli P, Bissoli F, et al. Bacterial infection in patients with advanced cirrhosis: a multicentre prospective study. Dig Liver Dis. 2001;33(1):41-8.

7. Wong F, Bernardi M, Balk R, Christman B, Moreau R, Garcia-Tsao G, et al. Sepsis in cirrhosis: report on the 7th meeting of the International Ascites Club. Gut. 2005;54(5):718-25.

8. Fernández J, Gustot T. Management of bacterial infections in cirrhosis. J Hepatol. 2012;56(1):1-12.

9. Abdel-Khalek EE, El-Fakhry A, Helaly M, Hamed M, Elbaz O. Systemic inflammatory response syndrome in patients with liver cirrhosis. Arab J Gastroenterol. 2011;12(4):173-7.

10. Bone RC, Balk RA, Cerra FB, Dellinger RP, Fein AM, Knaus WA, et al. Definitions for sepsis and organ failure and guidelines for the use of innovative therapies in sepsis. The ACCP/SCCM Consensus Conference Committee. American College of Chest Physicians/Society of Critical Care Medicine. Chest. 1992;101:1644-55.

11. Hurlimann J, Thorbecke GJ, Hochwald GM. The liver as the site of C-reactive protein formation. J Exp Med. 1966;123(2):365-78.

12. Oh BS, Jang JW, Kwon JH, You CR, Chung KW, Kay CS, et al. Prognostic value of C-reactive protein and neutrophil-to-lymphocyte ratio in patients with hepatocellular carcinoma. BMC Cancer. 2013;13:78.

13. An H, Jang J, Bae S, Choi J, Yoon S, Lee M, et al. Serum C-reactive protein is a useful biomarker for predicting outcomes after liver transplantation in patients with hepatocellular carcinoma. Liver Transpl. 2012;18(12):1406-14.

14. Papp M, Vitalis Z, Altorjay I, Tornai I, Udvardy M, Harsfalvi J, et al. Acute phase proteins in the diagnosis and prediction of cirrhosis associated bacterial infections. Liver Int. 2012;32(4):603-11.

15. Avanzas P, Quiles J, Lopez de Sa E, Sanchez A, Rubio R, Garcia E, et al. Neutrophil count and infarct size in patients with acute myocardial infarction. Int J Cardiol. 2004;97(1):155-6.

16. Halazun KJ, Aldoori A, Malik HZ, Al-Mukhtar A, Prasad KR, Toogood GJ, et al. Elevated preoperative neutrophil to lymphocyte ratio predicts survival following hepatic resection for colorectal liver metastases. Eur J Surg Oncol. 2008;34(1):55-60

17. Alkhouri N, Morris-Stiff G, Campbell C, Lopez R, Tamimi TA, Yerian L, et al. Neutrophil to lymphocyte ratio: a new marker for predicting steatohepatitis and fibrosis in patients with nonalcoholic fatty liver disease. Liver Int. 2012;32(2):297-302.

18. Leithead JA, Rajoriya N, Gunson BK, Ferguson JW. Neutrophil-to-lymphocyte ratio predicts mortality in patients listed for liver transplantation. Liver Int. 2015;35(2):502-9.

19. Tandon P, Garcia-Tsao G. Bacterial infections, sepsis, and multiorgan failure in cirrhosis. Semin Liver Dis. 2008;28(1):26-42.

20. Bernard B, Grange JD, Khac EN, Amiot X, Opolon P, Poynard T. Antibiotic prophylaxis for the prevention of bacterial infections in cirrhotic patients with gastrointestinal bleeding: a meta-analysis. Hepatology. 1999;29(6):1655-61.

21. Llach J, Rimola A, Navasa M, Gines P, Salmeron JM, Gines A, et al. Incidence and predictive factors of first episode of spontaneous bacterial peritonitis in cirrhosis with ascites: relevance of ascitic fluid protein concentration. Hepatology. 1992;16(3):724-7

22. Jang JW, Yoo SH, Kwon JH, You CR, Lee S, Lee JH, et al. Serum hepatitis B surface antigen levels in the natural history of chronic hepatitis $B$ infection. Aliment Pharmacol Ther. 2011;34(11-12):1337-46.

23. Bone RC, Balk RA, Cerra FB, Dellinger RP, Fein AM, Knaus WA, et al. Definitions for sepsis and organ failure and guidelines for the use of innovative therapies in sepsis. The ACCP/SCCM Consensus Conference Committee. American College of Chest Physicians/Society of Critical Care Medicine. Chest. 1992;101(6):1644-55.
24. Garcia-Tsao G. Bacterial infections in cirrhosis: treatment and prophylaxis. J Hepatol. 2005:42 Suppl 1:85-92.

25. Albillos A, de la Hera A, Gonzalez M, Moya JL, Calleja JL, Monserrat J, et al. Increased lipopolysaccharide binding protein in cirrhotic patients with marked immune and hemodynamic derangement. Hepatology. 2003;37(1):208-17.

26. Ijichi H, Taketomi A, Yoshizumi T, Uchiyama H, Yonemura $Y$, Soejima $Y$, et al. Hyperbaric oxygen induces vascular endothelial growth factor and reduces liver injury in regenerating rat liver after partial hepatectomy. J Hepatol. 2006;45(1):28-34

27. Plessier A, Denninger MH, Consigny Y, Pessione F, Francoz C, Durand F, et al. Coagulation disorders in patients with cirrhosis and severe sepsis. Liver Int. 2003;23(6):440-48.

28. Castell JV, Gomez-Lechon MJ, David M, Fabra R, Trullenque R, Heinrich PC. Acute-phase response of human hepatocytes: regulation of acute-phase protein synthesis by interleukin-6. Hepatology. 1990;12(5):1179-86.

29. Jang JW, Oh BS, Kwon JH, You CR, Chung KW, Kay CS, et al. Serum interleukin-6 and C-reactive protein as a prognostic indicator in hepatocellular carcinoma. Cytokine. 2012;60(3):686-93.

30. Cervoni JP, Thevenot T, Weil D, Muel E, Barbot O, Sheppard F, et al. C-reactive protein predicts short-term mortality in patients with cirrhosis. J Hepatol. 2012;56(6):1299-304.

31. Cho Y, Park SY, Lee JH, Lee DH, Lee M, Yoo JJ, et al. High-sensitivity C-reactive protein level is an independent predictor of poor prognosis in cirrhotic patients with spontaneous bacterial peritonitis. J Clin Gastroenterol. 2014;48(5):444-49.

32. Jimenez MF, Watson RW, Parodo J, Evans D, Foster D, Steinberg M, et al. Dysregulated expression of neutrophil apoptosis in the systemic inflammatory response syndrome. Arch Surg. 1997;132(12):1263-69.

33. Wesche DE, Lomas-Neira JL, Perl M, Chung CS, Ayala A. Leukocyte apoptosis and its significance in sepsis and shock. J Leukoc Biol. 2005;78(2):325-37.

34. Tritto G, Bechlis Z, Stadlbauer V, Davies N, Francés R, Shah N, et al. Evidence of neutrophil functional defect despite inflammation in stable cirrhosis. J Hepatol. 2011;55(3):574-81.

35. Mookerjee RP, Stadlbauer V, Lidder S, Wright GA, Hodges SJ, Davies NA, et al. Neutrophil dysfunction in alcoholic hepatitis superimposed on cirrhosis is reversible and predicts the outcome. Hepatology. 2007:46(3):831-40.

36. Tanriverdi H, Ornek T, Erboy F, Altinsoy B, Uygur F, Atalay F, et al. Comparison of diagnostic values of procalcitonin, C-reactive protein and blood neutrophil/lymphocyte ratio levels in predicting bacterial infection in hospitalized patients with acute exacerbations of COPD. Wien Klin Wochenschr. 2015. [Epub ahead of print]

37. Lowsby R, Gomes C, Jarman I, Lisboa P, Nee PA, Vardhan M, et al. Neutrophil to lymphocyte count ratio as an early indicator of blood stream infection in the emergency department. Emerg Med J. 2015;32:531-34

38. Liu $H$, Zhang $H$, Wan $G$, Sang $Y$, Chang $Y$, Wang $X$, et al. Neutrophillymphocyte ratio: a novel predictor for short-term prognosis in acute-on-chronic hepatitis B liver failure. J Viral Hepat. 2014;21:499-507.

39. Giron-Gonzalez JA, Martinez-Sierra C, Rodriguez-Ramos C, et al. Implication of inflammation-related cytokines in the natural history of liver cirrhosis. Liver Int. 2004;24(5):437-45.

40. Muller I, Munder M, Kropf P, Hansch GM. Polymorphonuclear neutrophils and T lymphocytes: strange bedfellows or brothers in arms? Trends Immunol. 2009;30(11):522-30.

41. Biyik M, Ucar R, Solak Y, Gungor G, Polat I, Gaipov A, et al. Blood neutrophilto-lymphocyte ratio independently predicts survival in patients with liver cirrhosis. Eur J Gastroenterol Hepatol. 2013;25:435-41. 